\title{
The Cooperative Anticancer Effect of Dual Styrenemaleic Acid Nano- Miceller System against Pancreatic Cancer
}

\author{
Khaled Greish ${ }^{1,2,3 *}$, Katherine Muller ${ }^{4}$, Julie IvanaJay ${ }^{2}$ and Don Haeng Lee ${ }^{2}$ \\ ${ }^{1}$ Department of Pharmacology \& Toxicology, Otago School of Medical Sciences, University of Otago, Dunedin, New Zealand \\ ${ }^{2}$ Utah-Inha DDS \& Advanced Therapeutics Research Center, Incheon, South Korea \\ ${ }^{3}$ Faculty of Medicine, Suez Canal University, Egypt \\ ${ }^{4}$ Faculty of Pharmaceutical Technology at Philipps-Universität, Marburg, Germany
}

\begin{abstract}
Pancreatic tumors remain one of the most formidable cancer types to treat. Patients with locally advanced or metastatic disease, which collectively represent over $80 \%$ of patients, rarely survive beyond one year. Pancreatic cancer is especially difficult to treat because of the uniqueness of its histology, with few vasculatures that can be used to supply anticancer agents. In this work we test a new system made of two styrene maleic acid (SMA) Nanomiceles encapsulating the phototosensetizer Zinc Protoporphyrin (ZnPP) or anticancer agent 4'-O-tetrahydropyranyl-doxorubicin (THP/Pirarubicin). Our hypothesis is that, local inflammatory response in tumor vessels induced by photosensitization can improve anticancer drug delivery and hence the anticancer activity. Two pancreatic tumor cell lines (Panc-1 and ASPC-1) were used to test the synergism of cytotoxic effect vitro. PANC-1 animal model in SCID mice were utilized to test the anticancer effect and the safety of the dual system in vivo. The photosensitizer activity of ZnPP nanomicelles followed by light irradiation and the administration of the anticancer micelles resulted in a synergetic antitumor effect in SCID mice in vivo but not in vitro. In conclusion, the dual Nanosystem of a photosensitizer and anticancer agent were well tolerated in animal model of pancreatic cancer and resulted in a synergistic anticancer effect. To our knowledge this is the first report of using a dual nanosystem of a photosensitizer and anticancer agent to treat pancreatic cancer.
\end{abstract}

\section{Introduction}

Pancreatic cancer has the worst mortality rate and the lowest overall survival in all cancers. Only $10 \%$ of patients are suitable for potentially curative surgery [1]. Even among those qualified for surgery, aggressive metastasis often occurs after surgery, which is highly resistant to chemotherapy and radiation therapy [2]. Chemotherapy is still the only option in metastatic pancreatic cancer treatment with minimal impact on survival. Gemcitabine (2'-2'-difluorodeoxycytidine) is the standard chemotherapy for all stages of pancreatic cancer. However, neither gemcitabine alone nor gemcitabine-based combinational chemotherapy achieves a favorable outcome in advanced disease [3].

One major limiting factor to the efficacy of anticancer drugs in pancreatic tumors is the presence of dense stromal component, challenging the diffusion and penetration of chemotherapeutic agents to the core of the tumor mass [4]. Further, pancreatic tumors tend to be hypo-vascular compared to other adenocarcinomas [5]. New strategies for treatment of pancreatic treatment are thus, highly needed. In this respect, the collaborative use of two Nano-micelles, each with different cellular target may provide efficacy against these inherently chemo resistant tumors.

We prepared two micelles based on styrene maleic acid (SMA) block copolymer encapsulating either the anthracycline; 4'-O-tetrahydropyranyl-doxorubicin (THP/Pirarubicin) or Zinc Protoporphyrin (ZnPP).

Pirarubicin(THP), a semi-synthetic derivative of doxorubicin, is a reactive oxygen species (ROS) generating agent with significant tumor growth inhibiting properties together with other molecular mechanisms for cell killing [6]. However, being a low molecular weight compound of $627 \mathrm{Da}$, it readily diffuses across normal as well as tumor blood vessels non-selectively. Its accumulation in the cardiac tissues results in ROS associated cardiac tissue damage. In contrast, Nanosize drug formulations can preferentially leak out of tumor vessels that are hyperpermeable. We have previously demonstrated that micellar formation of SMA copolymer encapsulating a high pay load of pirarubicin, up to $60 \% \mathrm{w} / \mathrm{w}$ [7]. SMA-THP have proven efficacy against various tumor models with excellent safety profile, due to its concentration in tumor tissues of more than 13 fold compared to native drug. $\mathrm{ZnPP}$ has inhibitory activity against heat shock protein 32 (HO-1) which is over expressed by pancreatic cancer cells and crucial to their survival [8]. ZnPP inhibits epidermal growth factor tyrosine kinase (EGFR-TK), associated with tumor progression and metastasis [9]. Further, and most relevant to current study approach, ZnPP poses photo sensitizer effect suited for photodynamic therapy (PDT) [10]. To enhance further the targeting capability of SMA-ZnPP micelles, we chemically conjugated glucosamine to the malate group in SMA backbone. Glucose conjugation can enhance tumor cell internalization through receptor-mediated endocytosis utilizing glucose receptor (GLU1), which is overexpressed by pancreatic tumor cells. The current work aims at examining the efficacy and safety of a new collaborative strategy involving dual SMA micelles against pancreatic tumors and if synergistic therapeutic advantages could be achieved by the combination therapy.

\section{Materials and Methods}

\section{Materials}

SMA-ZnPP and SMA-THP was prepared and kept as lyophilized

*Corresponding author: Khaled Greish, Adams building, 18 Frederick street, level 2 room 238, Tel: + 64- 3- 479- 4095; Fax: +64 -3-479-9140; Email: khaled. greish@otago.ac.nz

Received September 20, 2011; Accepted November 01, 2011; Published November 03, 2011

Citation: Greish K, Muller K, IvanaJay J, Lee DH (2011) The Cooperative Anticancer Effect of Dual Styrenemaleic Acid Nano-Miceller System against Pancreatic Cancer. J Nanomedic Nanotechnol S4:004. doi:10.4172/2157-7439. S4-004

Copyright: (C) 2011 Greish K, et al. This is an open-access article distributed under the terms of the Creative Commons Attribution License, which permits unrestricted use, distribution, and reproduction in any medium, provided the original author and source are credited. 
powder as described previously [11]. Samples were dissolved in phosphate buffered saline (PBS) and passed through $0.45 \mathrm{uM}$ filters before usage. Panc-1 and ASPC-1 human pancreatic cell line was kind gift from Inha university hospital, South Korea. Both cell lines were cultured in Advanced Roswell Park Memorial Institute (RPMI) 1640 media (Invitrogen, Carlsbad, CA) supplemented with $4 \mu \mathrm{M}$-glutamine and $10 \%(\mathrm{v} / \mathrm{v})$ fetal bovine serum (FBS).

\section{Preparation of SMA-ZnPP-GLU micelles}

To prepare SMA-GLU; $2.2 \mathrm{~mol}$ glucosamine and 2 mol sodium methoxide were dissolved in DMSO under completely dry conditions. After $3 \mathrm{~h}$ the resultant methanol was removed under vacuum at $20^{\circ}$ $60^{\circ} \mathrm{C}$. Then $1 \mathrm{~mol}$ SMA was added and the solution stirred for $12 \mathrm{~h}$. For dialysis $3.5 \mathrm{KDa}$ cut off membranes were used to remove the unreacted glucosamine. Then SMA-GLU was used at various feeding ratios to obtain SMA- ZnPP-GLU (Figure 1).

To measure the glucosamine content of SMA-ZnPP micelles, the samples (SMA-glucosamine, SMA-ZnPP-glucosamine) were dissolved in water to a final concentration of $1 \mathrm{mg} / \mathrm{ml}$. $25 \mu \mathrm{l}$ of each sample were mixed with $575 \mu \mathrm{l} \mathrm{HCl} 2 \mathrm{~N}$. Then they were heated in a boiling water bath for $16 \mathrm{~h}$. The samples and references were neutralized with $385 \mu \mathrm{l}$ $\mathrm{Na}_{2} \mathrm{CO}_{3}$ and $15 \mu \mathrm{l}$ Triethylamine to bind the salt of the solution. 500 $\mu \mathrm{l}$ of a $2 \%$ acetyl acetone in $1.5 \mathrm{M} \mathrm{Na}_{2} \mathrm{CO}_{3}$ solution were added and the mixture was heated in a boiling water bath for $20 \mathrm{~min}$. The mixture was cooled down and transferred into a more voluminous vial. $1 \mathrm{ml}$ Ethanol and $500 \mu \mathrm{l}$ Ehrlich's reagent were added. After $15 \mathrm{~min}$ the produced color was read at $530 \mathrm{~nm}$. Size measurement of the micelles was carried out by Dynamic light scattering (DLS) using Zeta sizer (nano series) from Malvern instruments (Malvern, UK) at a concentration of $1 \mathrm{mg} /$ $\mathrm{ml}$.

\section{In vitro anticancer activity}

In vitro cytotoxicity of SMA micelles was determined by means of the 3-(4, 5- dimethylthiazol-2-yl)-2, 5-diphenyltetrazolium bromide (MTT) assay with Panc-1 and ASPC-1 human pancreatic cancers. Cells were plated in 96- well culture plates (3000 cells/well). Cells were then cultured overnight in RPMI medium with $10 \%$ fetal calf serum. The cells were then incubated in the presence of different molar concentrations of THP, and ZnPP micelles for 3 days. Cell viability was measured by absorbance at $570 \mathrm{~nm}$ with $620 \mathrm{~nm}$ as reference using a multi-well plate<smiles>NC1C(O)OC(CO)C(O)C1O</smiles>

Glucosamine .

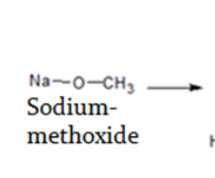
methoxide
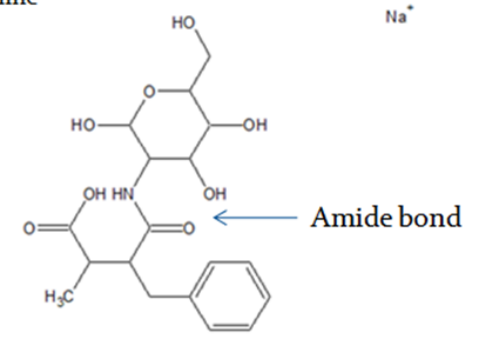

SMA

Figure 1: Chemical reaction of glucosamine with SMA by using sodium methoxide to form SMA-GLU miceller backbone. Different feeding ratio of the SMA-GLU was used to form SMA-ZnPP-GLU micelles. reader. Toxicity was quantified as the fraction of cells surviving relative to untreated controls.

\section{Cell propagation}

For establishing xenografts, PANC-1 cells were cultured in RPMI 1640 medium in 3 surface nunclon cell culture flasks (Nunclon, Roskilde, Denmark). Each flask have the yield of $30 \times 10^{6}$ Panc- 1 cells, a total of 35 flasks were used to propagate enough cells for animal studies.

\section{Light irradiation}

Light irradiation was performed utilizing ultraviolet lamp (osram Ultera-Vitalux $300 \mathrm{~W}$-Italy). The lamp emits light wavelength in the range of 270-420 nm. Both cells and animal were exposed to 50.000 Lux for 5 minutes to allow for interaction with SMA-ZnPP micelles. Light intensity was measured at the center of irradiation field by digital illuminometer (DX-200, INS, Taiwan).

\section{Animals}

6-8 male ddY mice were used to demonstrate the enhancement of drug delivery following SMA-ZnPP PDT. To test the efficacy and safety of the dual miceller system in pancreatic tumor model, 6 weeks male SCID mice were obtained from Korean research institute of bioscience and technology (KRIBB). Mice were kept in 12 hour light/ dark cycles under sterile conditions and were used in accordance with the Institutional Animal Care and Use Committee (IACUC) of Inha University.

\section{Demonstration of nanosize tumor drug enhancement in response to SMA-ZnPP}

To test the efficacy of SMA-ZnPP mediated PDT on improving the drug concentration in tumor tissue we used the putative Nanosize Evans blue dye (EBD) in S 180 tumors described as above. EBD was dissolved in DW and administered at $10 \mathrm{mg} / \mathrm{kg}$ of mice weight in 0.2 $\mathrm{ml}$, as the tumor reaches the diameter of $7 \mathrm{~mm}$. Thirty minutes after injection of SMA-ZnPP animals were irradiated with light as described earlier. At 6 hours following this treatment, Animals were humanly euthanized and the dorsal skin having the tumor was exposed and tumor extracted from the base using surgical scissor, weighed and added to $3 \mathrm{ml}$ formamide. Tumors from animals given EBD $(+/-)$ SMA-ZnPP PDT were harvested similarly. The tumors then were incubated at $60^{\circ} \mathrm{C}$ water bath with shaking for 48 hours to extract EBD. EBD concentrations were then quantified by absorbance at $620 \mathrm{~nm}$, and reading was converted into $\mu \mathrm{g} \mathrm{EBD} / \mathrm{mg}$ tissue using standard curve of EBD.

\section{In vivo studies}

To demonstrate the enhancement of drug delivery in response to SMA-ZnPP PDT, mouse fibrosarcoma model was used. Briefly; 2 million cells of mouse sarcoma (S-180) were implanted subcutaneously (s.c.) in the dorsal skin of the ddY mice. Animals were followed daily, and when tumors had reached an average diameter of $7 \mathrm{~mm}$ with no necrotic areas, treatment commenced. For evaluation of the collaborative anticancer efficacy of the dual miceller system, pancreatic cancer xenografts were established. Briefly, After tumor cell propagation described as above, cells were collected from Nunclon flasks by trypsinization and counted, then concentrated in ice cooled PBS at concentration of 5 million cells per $0.2 \mathrm{ml}$. Mice were then anesthetized using $4 \%$ isoflurane mixed with oxygen, by subcutaneously injecting $5 \times 10^{6}$ Panc- 1 cells bilaterally on the flank of each mouse. Tumors were allowed to grow for three weeks to reach average volume of 100 
$\mathrm{mm}^{3}$, then treatment commenced. Groups of five animals each with a total of ten tumors were randomly assigned to the study group. Table 1 shows the design of the study. Light irradiation was carried out for selected groups as described above. Animal were weighed and tumor size was measured with digital calipers biweekly. The estimated tumor volume $(V)$ was calculated using the longitudinal cross section $(L)$ and transverse cross section $(W)$ according to the formula: $V=(L \times W 2) / 2$ $\left(\right.$ expressed in $\left.\mathrm{mm}^{3}\right)$. The day of start of the treatments was designated as day 1 . To evaluate the MTD of SMA-THP, 3 mice were injected with doses up to $80 \mathrm{mg} / \mathrm{kg}$ with follow up of animal's weight for 2 weeks. The animal study design is shown in Table 1.

\section{Statistical analysis}

Differences between means were assessed by student $t$. test using Microsoft excel software. Data ware expressed as the mean \pm SE. Significant differences ware defined as $\mathrm{P}<0.05$.

\section{Results and Discussion}

\section{Synthesis and charcterixzartion of SMA-ZnPP-GLU micelles}

Tumor cells usually show a higher expression of glucosetransporter-protein (GLUT). GLUT-1 protein was found to be highly expressed in pancreatic cell culture as well as in isolated human pancreatic tumors $[12,13]$. By modification of SMA with glucosamine, we aimed at targeting this overexpression of transport proteins to increase the uptake of the micelle into the tumor cell. SMAglucosamine was synthesized as described in the methods. Figure 1 shows SMA-glucosamine chemical reaction. Using this reaction, we successfully attached 27.3 wt. \% glucosamine to SMA. As shown in Figure 2, the sizes of the micelle with different drug content and with or without conjugated glucosamine were close. The drug content and the attachment of glucosamine have no or only a very small influence on the size of the micelle. As true for other micelles, the size presented here is related to experimental condition (concentration, $\mathrm{pH}$, and time after preparation), verifying this parameter can result in different micelle conformation and thus different size [14]. Apart from slight difference in molecular size and charge, SMA-glucosamine micelles were similar to plain micelles prepared and described in [15]. The only significant difference between plain SMA-ZnPP and SMA-ZnPP-GLU was the higher release rate of SMA-GLU micelles. SMA-ZnPP and SMA-ZnPP-GLU release rate were $1.3 \%$ and $6 \%$ per day respectively at buffered phosphate saline with $\mathrm{pH}$ 7.0. The modification of SMA with glucosamine could have resulted in decreased stability of the micelle and lead to a higher release rate. A reason for that could be the core/ shell ratio change, or the hydrophilic hydrophobic shift towards the hydrophilic side, both factors might have contributed to this improved release profile, however, these remains to be investigated. The current improvement of release profile can prove to be beneficial in delivering

\begin{tabular}{|l|l|l|l|}
\hline Treatment & Dose $\mathbf{~ m g / k g}$ & N & Total \\
\hline Control & NA & 5 & 5 \\
\hline SMA-ZnPP (15\%) + light & 4,8 & 5 & 10 \\
\hline SMA-ZnPP (15\%) Glu & 4,8 & 5 & 10 \\
\hline SMA-ZnPP (15\%) Glu + light & 4,8 & 5 & 10 \\
\hline SMA-THP & 10,20 & 5 & 10 \\
\hline SMA-THP + SMA-ZnPP + light & 4,10 & 5 & 5 \\
\hline SMA-THP + SMA-ZnPP & 4,10 & 5 & 5 \\
\hline SMA-THP + SMA-ZnPP (Glu) + light & 4,10 & 5 & 5 \\
\hline Total 60 & & & \\
\hline
\end{tabular}

Table 1: Animal study groups. the active $\mathrm{ZnPP}$ to tumor cells that cannot be reached through the vascular access. Drug released from micelle and diffusing into tumor cells can further the photodynamic effect of ZnPP photosensitization deeper into tumor tissues. This might be even more advantageous with tumor of special nature such as the pancreatic tumors that have limited vascular access.

\section{In vitro activity of the dual SMA miceller system against panceritic tumor cell lines}

Panceriatic tumors are knowen for its inherent resistance for chemotherapy [2]. Gemcitabine (2', 2'-difluorodeoxycytidine) is the standard treatment for patients with advanced pancreatic cancer. However, gemcitabine has shown modest response rates (partial and complete responses) of $6-11 \%$ with disease stabilization occurring in a further 19-32\% [16,17]. This challenging resistance of pancreatic tumors warranted the proposed combination therapy evaluated in this study. The SMA-ZnPP photosensitizer was used prior to the administration of the chemotherapeutic cytotoxic anthracyclin micelles. As shown in Figure 3, ZnPP micelles showed higher anticancer activity against Panc1 cells compared to ASPC-1, further, in both cell lines, light irradiation for 5 minutes at 50000 Lux improves the cytotoxicity of micelles. Even though the improvement of cytotoxicity with PDT was $15-20 \%$ over no light irradiation, this limited improvement noticed in this study on tested pancreatic cell lines is reproducible and in accordance with previous studies both in vitro and in vivo $[10,11]$.

A

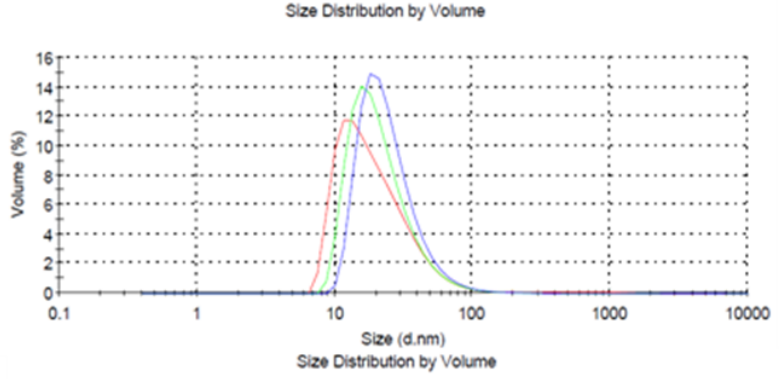

B

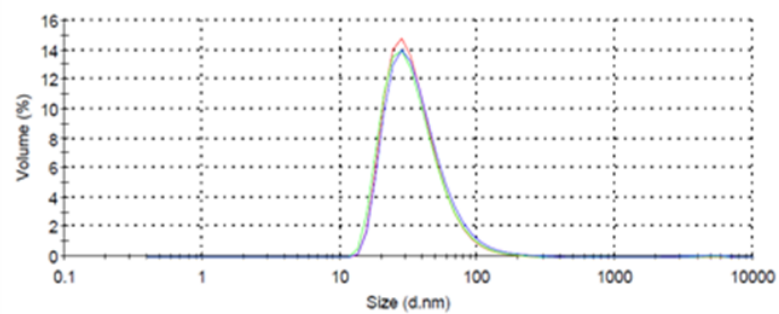

C

Size Distroution by Volume

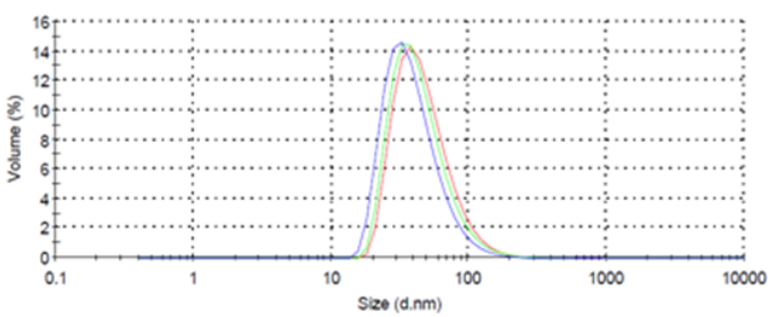

Figure 2: The size distribution of different SMA-ZnPP micelles. Three independent measurements were done for each loading at the concentration of $1 \mathrm{mg} / \mathrm{ml}$ in DW within $10 \mathrm{~min}$ after dissolution. The average size of A. SMA$\mathrm{ZnPP} 15 \%$ is $54.6 \mathrm{~nm}$; B. SMA- ZnPP-GLU $15 \%$ is $54.7 \mathrm{~nm}$; and C. SMA$\mathrm{ZnPP} 30 \%$ is $62.0 \mathrm{~nm}$. 
The presence of anticancer activity in the dark in response to SMA-ZnPP can be attributed to the Heme oxygenase-1 (HO-1) inhibition activity of $\mathrm{ZnPP}$. HO-1 has been repeatedly reported to be overexpressed in human pancreatic tumors especially in response to gemcitabine or radiation [8]. Further HO-1 over expression in animal pancreatic models, were shown to accelerate tumor aggressiveness, by increasing tumor growth, angiogenesis and metastasis in mice. The mere inhibition of HO-1 with SMA-ZnPP thus can account for the significant cytotoxic effect noted in the pancreatic cell lines in the absence of the PDT effect [18]. The enhancement of the cytotoxicity of SMA-ZnPP by light irradiation in both pancreatic cell lines is a further proves of the suitability of $\mathrm{ZnPP}$ micelles for Photodynamic therapy as previously demonstrated [11]. Both cell lines show relative resistance against SMA-THP anthracyclein micelle, though comparable to that of gemcitabine. Combination of SMA-THP and SMA-ZnPP micelles did not show added benefit to the efficacy of either of them (data not shown). The reason of this result in vitro was expected, as the main purposed mechanism of SMA-ZnPP enhancement was thought to be related to the enhancement of the delivery of the second agent (SMATHP) through the resultant local inflammation on the tumor vessels that support the growing tumor. With such element lacking from the in vitro experimental conditions, verification of our research hypothesis was only possible with the in vivo animal experiments. SMA-ZnPPGLU showed similar cyctoxic activity compared to the plain SMAZnPP micelles (data not shown). Possible explanation of such result could be related to the in vitro cytotoxicity assay condition where the glucose level in the culture media is several folds of the normal glucose level in mice or human plasma in biological environment. The presence of high glucose level can thus compete with SMA-GLU for the GLUT-1 receptors efficiently in vitro. In contrast, in vivo tumor environment usually have limited glucose availability, this render targeting tumor cells with this strategy a plausible strategy.

\section{Enhancement of nanomedicine drug delivery after SMA- ZnPP PDT}

In photodynamic therapy, typically a photosensitizer is administrated systemically or locally followed by light photoactivation, leading to the generation of cytotoxic reactive oxygen species in the presence of oxygen.

PDT as such has been linked to enhanced tumour vascular permeability $[19,20]$. In one study, it was found that the concentration of $2000-\mathrm{kDa}$ FITC-dextran were 5-folds higher in orthotropic MatLylu rat prostate tumors treated with vascular-targeting photodynamic therapy verteporfin, at $15 \mathrm{~min}$ following light irradiation, compared to non-irradiated control group [21]. The permeability enhancement verteporfin photosensitization was attributed to its effect on endothelial cell morphology, and cytoskeleton. Photosensitization was found to causes endothelial cell microtubule depolymerisation and induces the formation of actin stress fibres. Thus endothelial cells were found to retract, leading to the formation of intercellular gaps, which result in enhanced vascular permeability. In addition, endothelial cell damage leads to the establishment of thrombogenic sites within the vessel lumen and this initiates a physiological cascade of responses including platelet aggregation, the release of vasoactive molecules, leukocyte adhesion and increases in vascular permeability [20]. Based on the previous studies, we tested and quantified the enhancement of EBD delivery to S-180 tumors in ddY mice. EBD instantly complex with plasma albumin resulting in a Nanosize molecular complex, which can simulate the concentration of related Nanosize molecules in tumour tissues. As shown in Figure 4, the administration of SMA-ZnPP followed by light irradiation resulted in three folds higher increase in the concentration in EBD in similar tumour volume compared to animals which did not receive prior PDT. In addition, in Figure $4 \mathrm{~b}$, the local inflammatory response in vasculature in response to PDT could be clearly noticed.

\section{In vivo anticancer activity of SMA -micelles against Panc-1}

Pirarubicin (THP) is a pyranyl derivative of doxorubicin, its mechanism of action involves the generation of reactive oxygen species. THP is characterized by faster intracellular uptake and more potent antitumor activity than doxorubicin in vivo due to its pyranyl group and lipophilic properties. However, it exerts poor tumor targeting properties as is common to other low molecular weight anticancer agents. Once SMA-THP micelles reach the target tumor tissue, the micelles will release free pirarubicin slowly in a sustained manner in the vicinity of tumor cells that can even further into the
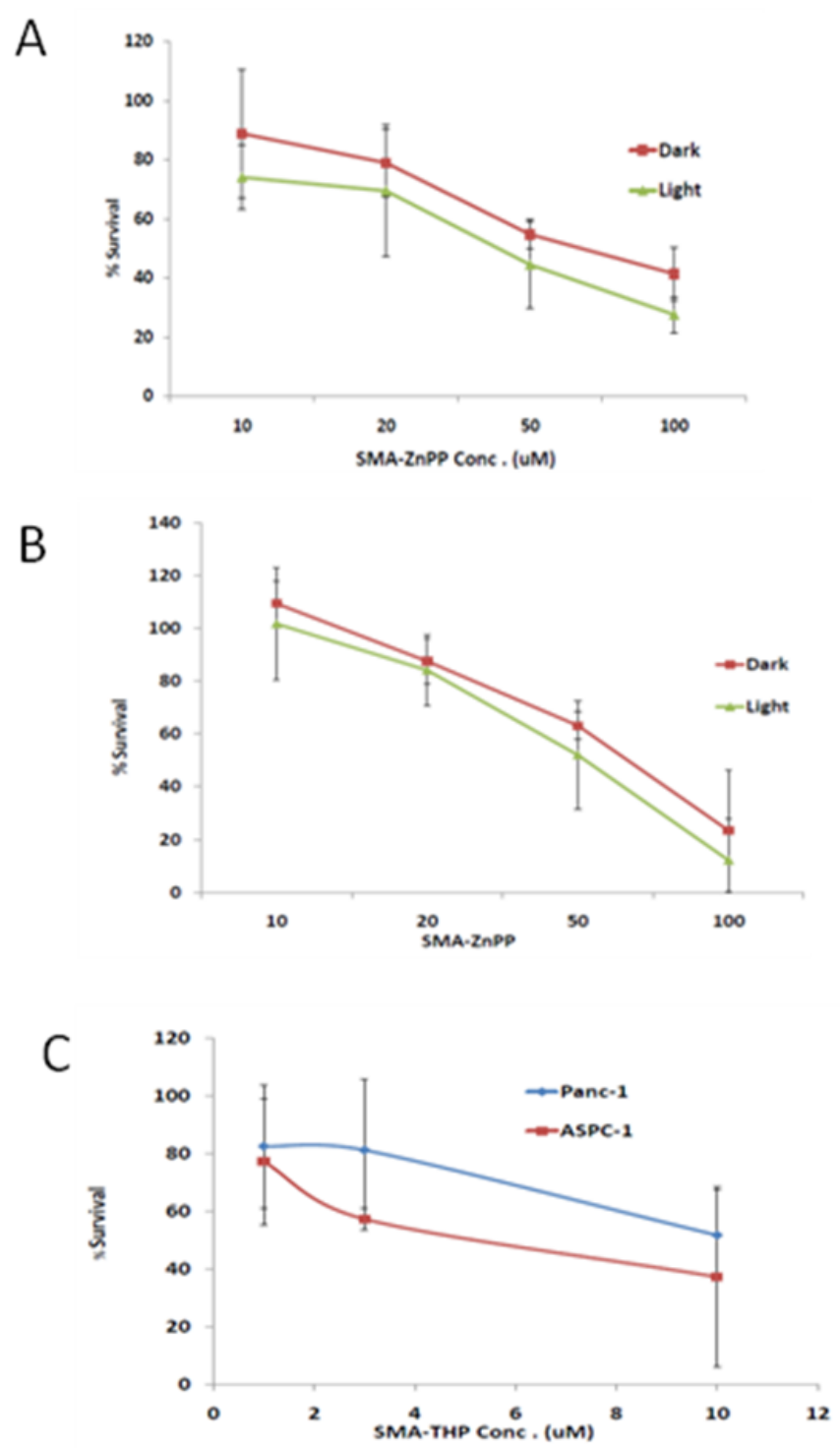

Figure 3: In vitro anticancer activity of SMA-micelles against Panc-1, and ASPC-1. (A) The effect of SMA-ZnPP against ASPC-1 with/without 50000 Lux irradiation for 5 minutes (B) The effect against PANC-1 pancreatic cell line. C. The effect of SMA-THP micelles in both cell lines 
A

$\mathrm{C}$
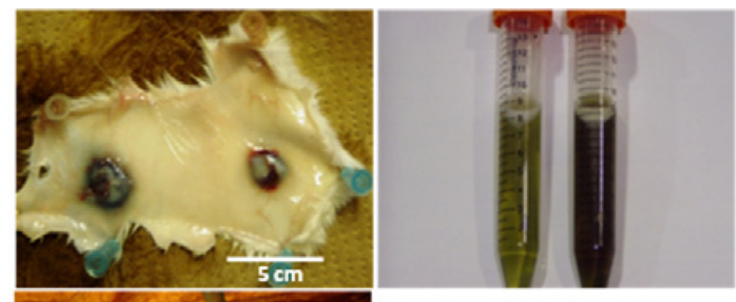

B
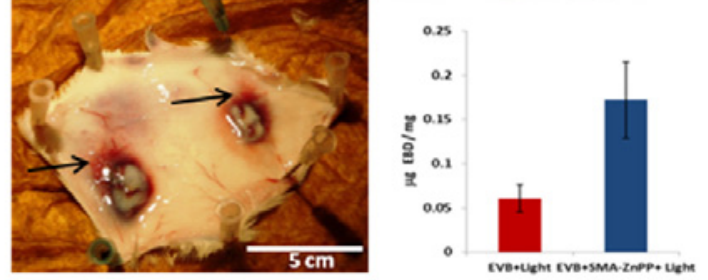

Figure 4: Enhancement of EBD tumor permeability in response to SMA ZnPP photosensitization. (A) EBD concentration in S-180 murine sarcoma without PDT (B) After injection of $5 \mathrm{mg} / \mathrm{kg}$ ZnPP photosensitizer, followed by 5 minutes light irradaition at 50.000 Lux. The arrows point to areas of vascular inflammation, scale bar: $5 \mathrm{~cm}$. (C) Representative tubes of the extracted EBD from light irradiated and non-irradiated animals. (D) The difference between extracted EBD with light irradiation was almost 3 folds higher compared to no irradiation.

pancreatic tumour tissue owing to the vascular inflammation mediated by the PDT effect. In our previous work, we have shown significant therapeutic effect of SMA-pirarubicin accompanied by up to 13 fold higher concentration of the THP micelle in tumour tissues compared to free THP. Among the two pancreatic cell lines used in this study (i.e. with Panc-1 and ASPC-1), Panc-1 cells showed higher take and better tumor development in SCID mice in a limited trial. SMA-THP was dosed at either 10 or $20 \mathrm{mg} / \mathrm{kg}$ animal weight. As shown in Figure $5 \mathrm{~A}$, both concentration of administered SMA-THP micelle showed significant tumor inhibition which is dose dependent. In addition, adding SMA-ZnPP further increased the anticancer efficacy (contrary to the in vitro data). The difference between the treated groups and control group was statistically significant. Figure $5 \mathrm{~B}$ shows the difference between the SMA-ZnPP micelles at 4 , and $8 \mathrm{mg} / \mathrm{kg}(+/-)$ light irradiation. The micelle shows higher anticancer activity with light irradiation, the addition of glucose moiety showed enhanced activity at $8 \mathrm{mg} / \mathrm{kg}$ compared to the non-targeted micelles.

\section{Safety of the dual miceller system in vivo}

Animal weights were measured twice a week as an indicator of general toxicity during the efficacy study. Relative to untreated animals, animals treated with SMA- micelles showed no significant difference in normalized mean animal weight during the study. Matter of fact, the control group showed high weight reduction compared to all micelle treated groups, even considering the extra weight added due to tumour progression in control group. These results demonstrate clearly that SMA- micelles were well tolerated (Figure 6). It worth mentioning that in a small study involving 3 animals, the animals tolerated the injection of SMA-THP at $80 \mathrm{mg} / \mathrm{Kg}$ in sequential injections, with less than $10 \%$ weight loss over 2 week's duration (data not shown ).

Thus our data proves that collaborative system used in this study, not only an effective strategy to tackle pancreatic cancer but a remarkably safe system as well.

\section{Conclusion}

A dual miceller system of SMA-ZnPP and SMA-THP was designed
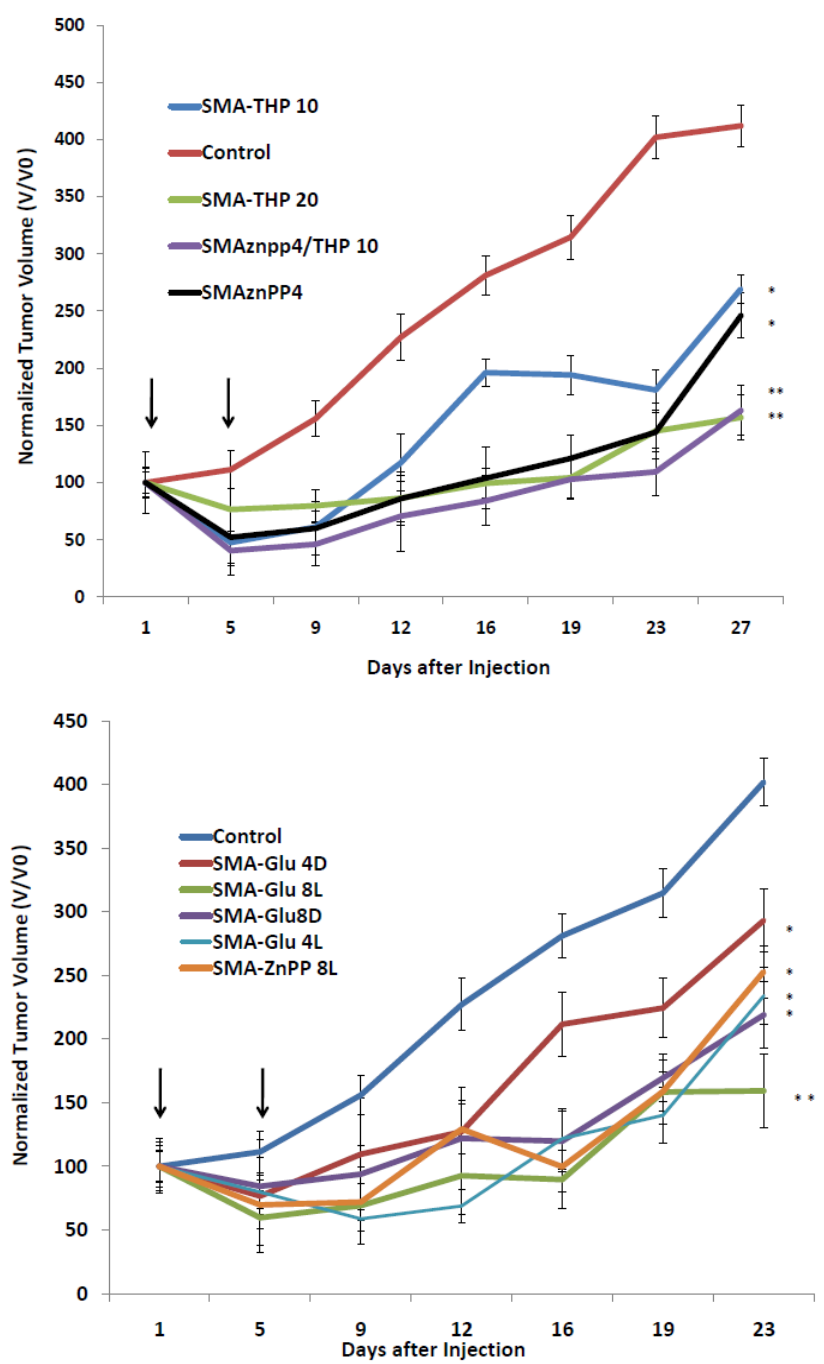

Figure 5: In vivo anticancer activity of SMA micelles against Panc-1 pancreatic cell lines in SCID mice. (A) The effect of SMA- THP at 10 , and $20 \mathrm{mg} / \mathrm{kg}$ as well as in addition to SMA-ZnPP with 50.000 Lux irradiation for 5 minutes (B) Comparison between glucose targeted SMA-ZnPP micelles to plain micelles.

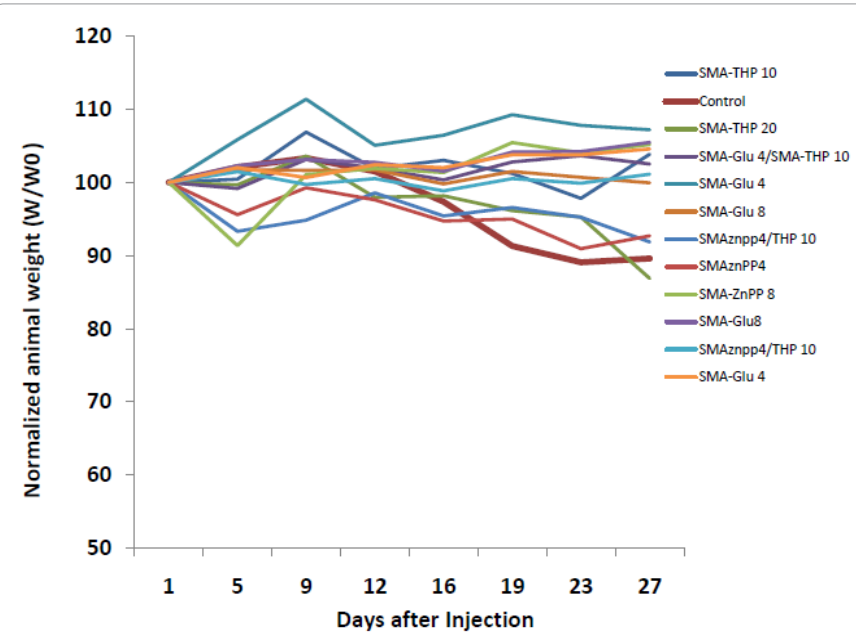

Figure 6: The effect of all study compounds on animal weigh. Arrow, days of injection/light irradiation, D; Dark, L; light. ${ }^{* *}, P$ value $<0.01,{ }^{*} P<0.05$. 
Citation: Greish K, Muller K, IvanaJay J, Lee DH (2011) The Cooperative Anticancer Effect of Dual Styrenemaleic Acid Nano-Miceller System against Pancreatic Cancer. J Nanomedic Nanotechnol S4:004. doi:10.4172/2157-7439.S4-004

and tested in treatment of pancreatic cancer animal model. The two miceller system proved synergistic effect against Panc -1 tumor pancreatic tumor model in SCID mice. Possible mechanism for the results obtained could be related to the vascular inflammatory response elicited by SMA-ZnPP photo irradiation, which clearly shown threefold increase in EBD concentration in tumor tissues. To our Knowledge, this is the first report that describes a dual collaborative Nano systems involving PDT for treatment of pancreatic tumors.

\section{Acknowledgements}

This work has been supported by funded by IFEZ research grant to Utah-Inha DDS center, and Departmental fund No.; (PL. 108403.01.S. LM) to Khaled Greish from department of pharmacology and toxicology, Otago KG thanks Professor Hiroshi Maeda for the kind gift of THP. KG thanks Sungeun Yang and YoonHo Jang for their help with animal studies.

\section{References}

1. Jemal A, Murray T, Samuels A, Ghafoor A, Ward E, et al. (2003) Cance statistics. CA Cancer J Clin 53: 5-26.

2. Yeo CJ, Cameron JL, Sohn TA, Lillemoe KD, Pitt HA et al. (1997) Six hundred fifty consecutive pancreaticoduodenectomies in the 1990s: pathology, complications, and outcomes. Ann Surg 226: 248-257.

3. Hawes RH, Xiong Q, Waxman I, Chang KJ, Evans DB, et al. (2000) A multispecialty approach to the diagnosis and management of pancreatic cancer. Am J Gastroenterol 95: 17-31.

4. Camps JL, Chang SM, Hsu TC, Freeman FR, Hong SJ, et al. (1990) Fibroblastmediated acceleration of human epithelial tumor growth in vivo. Proc Natl Acad Sci U S A 87: 75-79.

5. Ikeda N, Adachi M, Taki T, Huang C, Hashida H, et al. (1999) Prognostic significance of angiogenesis in human pancreatic cancer. $\mathrm{Br} \mathrm{J}$ Cancer 79 : 1553-1563.

6. Enomoto K, Abe O, Tominaga T, Abe R, Lino Y, et al. (1990) Clinical study of pirarubicin for breast cancer in Japan. Clinical Study Group of THP for Breast Cancer in Japan. Am J Clin Oncol 13: 48-53.

7. Greish K, Nagamitsu A, Fang J, Maeda H (2005) Copoly(styrene-maleic acid)pirarubicin micelles: high tumor-targeting efficiency with little toxicity. Bioconjug Chem 16: 230-236.

8. Berberat PO, Dambrauskas Z, Gulbinas A, Giese T, Giese N, et al. (2005)
Inhibition of heme oxygenase- 1 increases responsiveness of pancreatic cancer cells to anticancer treatment. Clin Cancer Res 11: 3790-3798.

9. Ritter CA, Arteaga CL (2003) The epidermal growth factor receptor-tyrosine kinase: a promising therapeutic target in solid tumors. Semin Oncol 30: 3-11.

10. Iyer AK, Greish K, Seki T, Okazaki S, Fang J, et al. (2007) Polymeric micelles of zinc protoporphyrin for tumor targeted delivery based on EPR effect and singlet oxygen generation. J Drug Target 15: 496-506.

11. Regehly M, Gresih K, Rancan F, Maeda H, Bohm F, et al. (2007) Water-soluble polymer conjugates of ZnPP for photodynamic tumor therapy. Bioconjug Chem 18: 494-499.

12. Macheda ML, Rogers S, Best JD (2005) Molecular and cellular regulation of glucose transporter (GLUT) proteins in cancer. J Cell Physiol 202: 654-662.

13. Medina RA, Owen GI (2002) Glucose transporters: expression, regulation and cancer. Biol Res 35: 9-26.

14. Yokoyama M, Satoh A, Sakurai Y, Okano T, Matsumura Y, et al. (1998) Incorporation of water-insoluble anticancer drug into polymeric micelles and control of their particle size. J Control Release 55: 219-229.

15. Iyer AK, Greish K, Fang J, Murakami R, Maeda H (2007) High-loading nanosized micelles of copoly(styrene-maleic acid)-zinc protoporphyrin for targeted delivery of a potent heme oxygenase inhibitor. Biomaterials 28: 18711881.

16. Carmichael J, Fink U, Russell RC, Spittle MF, Harris AL, et al. (1996) Phase II study of gemcitabine in patients with advanced pancreatic cancer. $\mathrm{Br} \mathrm{J}$ Cancer 73: $101-105$.

17. Casper ES, Green MR, Kelsen DP, Heelan RT, Brown TD, et al. (1994) Phase II trial of gemcitabine (2,2'-difluorodeoxycytidine) in patients with adenocarcinoma of the pancreas. Invest New Drugs 12: 29-34.

8. Sunamura M, Duda DG, Ghattas MH, Lozonschi L, Motoi F, et al. (2003) Heme oxygenase-1 accelerates tumor angiogenesis of human pancreatic cancer. Angiogenesis 6: 15-24

19. Dougherty TJ, Gomer CJ, Henderson BW, Jori G, Kessel D, et al. (1998) Photodynamic therapy. J Natl Cancer Inst 90: 889-905.

20. Fingar VH (1996) Vascular effects of photodynamic therapy. J Clin Laser Med Surg 14: 323-328.

21. Chen B, Pogue BW, Luna JM, Hardman RL, Hoopes PJ, et al. (2006) Tumo vascular permeabilization by vascular-targeting photosensitization: effects, mechanism, and therapeutic implications. Clin Cancer Res 12: 917-923.

\footnotetext{
This article was originally published in a special issue, Nanotechnology: Targeted Drug Delivery handled by Editor(s). Dr. Sami M. Nazzal, University of Louisiana at Monroe, USA; Dr. Kytai Troung Nguyen, University of Texas at Arlington, USA
} 